\title{
Valeur nutritionnelle des viscères et branchies issus du nettoyage du poisson Pseudotolithus typus dans les marchés de Douala
}

\author{
P. R. N. NDÔMBÔL ${ }^{2}$, J. M. NJINKOUE ${ }^{1 *}$, S. R. MOUOKEU ${ }^{2}$, J. B. DJOPNANG ${ }^{2}$, \\ J. C. MANZ KOULE ${ }^{1}$, C. S. MILONG MELONG ${ }^{2}$, M. N. NCHOUTPOUEN ${ }^{1}$ et \\ F. TCHOUMBOUNGNANG ${ }^{2}$
}

\author{
${ }^{1}$ Laboratoire de Sciences Alimentaires et Nutrition, Département de Biochimie, Faculté des Sciences, \\ Université de Douala, B.P. 24157 Douala, Cameroun. \\ ${ }^{2}$ Laboratory of Fisheries \& Aquatic resources, Institute of Fisheries \& Aquatic Sciences at Yabassi, University \\ of Douala, Po Box 7236 Douala, Cameroon. \\ *Auteur correspondant ; E-mail : jmnjinkoue@yahoo.fr
}

\section{RESUME}

L'étude de la valeur nutritionnelle des sous-produits de traitement de poisson contribue à la valorisation des déchets rejetés dans l'environnement. La détermination de la valeur nutritionnelle des viscères et branchies issus du poisson de l'espèce Pseudotolithus typus a été réalisée. L'analyse bromatologique a été réalisée selon les méthodes standards. Les huiles extraites de ces sous-produits ont été caractérisées par la détermination des indices. Dix-huit rats adultes répartis en trois lots de six chacun ont reçu différents régimes équilibrés respectivement Régime Standards, possédant la farine de poisson à 10\%, Régime Alimentaire (substitution des $10 \%$ de la farine de poisson par la farine des sous-produits de P. typus) et le Régime Neutre (farines précédentes remplacées par les farines végétales). L'estimation de ces sous-produits rejetés dans l'environnement est de $12,55 \%$ de la masse du poisson entier. La farine des sous-produits montre une teneur de 20,33 $\pm 0,57 \%$ d'humidité; $59,81 \pm$ $0,17 \%$ de protéines; $24,33 \pm 0,57 \%$ de cendres; $15,11 \pm 0,05 \%$ de lipide ; $0,75 \pm 0,46 \%$ de glucides totaux, et des éléments minéraux tels que, le calcium $(4,63 \pm 0,42 \mathrm{~g} / \mathrm{kg})$, le phosphore $(5,59 \pm 0,45 \mathrm{~g} / \mathrm{kg})$, et le fer $(323 \pm 18,36 \mathrm{mg} / \mathrm{kg})$. Les indices étudiés sont compris dans l'intervalle des valeurs de la norme du codex alimentarius, avec un indice d'iode élevé $122,735 \pm 0,915 \mathrm{mg} / \mathrm{kg}$. Le régime alimentaire reçu par les rats est plus efficace que le régime neutre en référence à la matière sèche totale ingérée et la différence de gain du poids. Il est plus digeste que le régime "RS" en référence à la digestibilité apparente et réelle. Les viscères et branchies de $P$. typus ont un potentiel nutritionnel élevé et peuvent être utilisés comme complément alimentaire pour bétail. (C) 2019 International Formulae Group. All rights reserved

Mots clés : Pseudotolithus typus, sous-produits, valeurs nutritionnelles, Rattus norvegicus.

\section{Nutritional value of the viscera and gills resulting from the cleaning of the Pseudotolithus typus fish in the Douala markets}

\begin{abstract}
The study of the nutritional value of fish processing by-products contributes to the recovery of waste released into the environment. We carried out the determination of the nutritional value of viscera and gills from Pseudotolithus typus fish. Bromatological analysis was performed according to standard methods. Oils extracted
\end{abstract}


from these by-products have been characterized by the determination of theirs indices. Eighteen adult rats were divided into three batches of six. Each received different balanced diets respectively Standard Diet containing $10 \%$ fish meal, Test Diet (substitution of $10 \%$ of fish meal by the flour of the by-products of $P$. typus) and Neutral Diet (fish flours replaced by plant flours). These released by-products were estimated to be about $12.55 \%$ of the total fish mass. Moisture content of their flour was $20.33 \pm 0.57 \% ; 59.81 \pm 0.17 \%$ protein; $24.33 \pm 0.57 \%$ ash; $15.11 \pm 0.05 \%$ lipid; $0.75 \pm 0.46 \%$ total carbohydrates. For mineral elements, calcium stands for $4.63 \pm 0.42$ $\mathrm{g} / \mathrm{kg}$, phosphorus $5.59 \pm 0.45 \mathrm{~g} / \mathrm{kg}$, and iron $323 \pm 18.36 \mathrm{mg} / \mathrm{kg}$. Indices were within the range of values performed in codex alimentarius standard. Iodine number was high $(122.735 \pm 0.915 \mathrm{mg} / \mathrm{kg})$. Test diet received by rats were more effective than the neutral diet when considering the total dry matter ingested and weight gain. It is more digestible than the standard diet with respect to apparent and actual digestibility. Therefore, viscera and gills of $P$. typus have high nutritional potential and can be used as a dietary supplement for livestock.

(C) 2019 International Formulae Group. All rights reserved

Keywords: Pseudotolithus typus, by-products, nutritional value, Rattus norvegicus.

\section{INTRODUCTION}

La production mondiale des produits halieutiques est estimée à 171 millions de tonnes, soit 20,5 Kg/habitant/an (FAO, 2016). Les différents traitements effectués sur ces tonnes de produit halieutique avant la consommation, produisent de grandes quantités de sous-produits sous formes de têtes, branchies, viscères, arêtes, queues, nageoires, écailles et peaux. Ils représentent entre 30 et $60 \%$ du poids de l'animal et proviennent du filetage, de l'éviscération, de l'étêtage, du pelage, du lavage, de la décongélation ou de la cuisson (Anaïs, 2014). Dans les pays industrialisés, les sous-produits issus du poisson sont transformés en aliments pour animaux (Khalifa, 2011). Au Cameroun, les parties des poissons tels que les têtes, queues et peaux ne sont pas considérées comme des sous-produits car elles sont consommées par les populations. Ceux des parties considérées comme sous-produits sont rejetées dans la nature, ce qui contribue à la pollution de l'environnement. Ainsi, dans le but de valoriser les sous-produits de la pêche, de contribuer à la lutte contre la prolifération des déchets dans l'environnement, nous nous sommes proposés de déterminer la valeur nutritionnelle des viscères et branchies prélevées sur l'espèce Pseudotolithus typus. L'objectif général étant de déterminer la composition chimique et la valeur nutritionnelle des viscères et branchies issus du nettoyage du poisson de l'espèce Pseudotolithus typus. Il s'agit pour nous de :

- Effectuer un essai d'estimation de la quantité de sous-produits issus du nettoyage de ce poisson ;

- Déterminer la composition chimique des sous-produits et caractériser les huiles extraites;

- Evaluer la biodisponibilité de ces sousproduits chez les rats.

\section{MATERIEL ET METHODES}

Les mesures morpho-métriques ont été prises pour l'ensemble des poissons (Pseudotolithus typus) source de nos sousproduits. Les sous-produits ont été prélevés, nettoyés et conservés à $-20^{\circ} \mathrm{C}$ jusqu'à leur utilisation. Ils ont été séchés dans l'étuve à une température de $55^{\circ} \mathrm{C}$ pendant un temps moyen de $15 \mathrm{~h}$. Ils ont été refroidis et écrasés à l'aide d'un robot de cuisine pour obtenir une poudre de couleur jaune.

\section{Etude in vitro des viscères et branchies}

Les teneurs en humidité, en cendres, en minéraux (Dosage du $\mathrm{Ca}, \mathrm{Mg}, \mathrm{K}, \mathrm{Na}, \mathrm{P}, \mathrm{Zn}$, $\mathrm{Cu}, \mathrm{Mn}$ et $\mathrm{Fe}$ ), en protéines totales (Dosage de l'azote total par la méthode de Kjeldahl) ont été obtenues par les méthodes de l'AOAC (1990) et les Glucides Totaux par la méthode de différence. 
Les teneurs en lipides ont été déterminées par extraction au Soxhlet (UIPAC, 1981). Les lipides extraits ont été caractérisés par la détermination des indices de peroxyde, d'anisidine, d'acide, d'iode (AFNOR, 1981) et d'oxydation totale: TOTOX (Shahidi et Miraliakbari, 2008).

\section{Etude in vivo des viscères et branchies Echantillonnage des animaux}

Dix-huit rats mâles albinos de souche Wistar (Rattus norvegicus) âgés de 45 à 60 jours pesant en moyenne $150 \pm 1,07 \mathrm{~g}$ ont été utilisés.

Les dix-huit rats ont été répartis en trois lots de 6 rats. Le lot 1 a reçu un régime standard (RS) contenant $10 \%$ de la farine de poisson vendu au niveau des provenderies; le lot 2 soumis au régime alimentaire (RA) est la substitution des $10 \%$ de la farine de poisson par la poudre des sous-produits de P.typus et le lot 3 ou régime neutre (RN) est la substitution des farines précédentes par les farines végétales. Les différents régimes constitués sont indiqués dans le Tableau 1.

Ces régimes au moment de leur distribution aux rats, ont été transformés en pâte ou en purée afin de minimiser les pertes

L'expérience a été réalisée pendant 16 jours avec une période d'acclimatation de deux jours et une période de croissance de 14 jours, durant lesquels les animaux ont été nourris à l'aliment standard suivi des différents régimes. Les cinq derniers jours de la période d'expérimentation ont servi à la mesure des bilans azotés (Meite et al., 2008 ; Bouafou et al., 2011).

La matière sèche de chaque régime a été mesurée quotidiennement. Le lendemain, avant d'effectuer la distribution, les refus (restes et gaspillages) sont collectés et leur matière sèche déterminée.

De l'eau propre, renouvelée fréquemment, les aliments aussi sont donnés à volonté aux animaux. Les quantités d'aliments consommées sont obtenues par la différence entre les quantités distribuées et les quantités refusées.

Les animaux sont pesés en début d'expérience puis à intervalle de deux jours; la dernière pesée a lieu à la fin de la période expérimentale.

Pendant la période du bilan azoté, des mesures de consommation, la collecte des fèces et des urines ont été faite quotidiennement par rat et par lot. Les fèces prélevées ont été pesées, séchées à l'étuve à $70^{\circ} \mathrm{C}$ pendant $24 \mathrm{~h}$, broyées puis mélangées pour la détermination de l'azote total. Les urines ont été collectées, pesées et additionnées de quelques gouttes d'acide chlorhydrique à $0,1 \mathrm{~N}$ pour la détermination de l'azote total.

Les paramètres d'étude de la valeur nutritionnelle sont consignés dans le Tableau 2.

\section{ANALYSES STATISTIQUES :}

L'analyse statistique des données recueillies s'est faite à l'aide des logiciels GraphPad InStat, Past et IBM SPSS Stastistc 20. Le PLSD (Protected Least Significant difference) de Fisher (test de comparaison post hoc) et Student-Newman-Keuls Multiple Comparisons Test ont été utilisés pour faire les comparaisons deux à deux entre les paramètres de la valeur nutritionnelle obtenus lorsque la pvalue de l'analyse des variances (ANOVA) était significative. Le seuil de signification a été fixé à une valeur de probabilité $\leq 0,05$. 
Tableau 1 : Formulation des ratios pour chaque lot de rat.

\begin{tabular}{lllll}
\hline Ingrédient (g) & \% de la matière sèche Ms & RS(g) & RA $(\mathbf{g})$ & RN $(\mathbf{g})$ \\
\hline Farine de poisson & $10 \%$ & 100 & & \\
Coproduit & & & 100 & \\
Maïs & $55 \%$ & 550 & 550 & 625,34 \\
Remoulage & $5 \%$ & 50 & 50 & 50 \\
Soja & $18 \%$ & 180 & 180 & 204,66 \\
Concentré & $1 \%$ & 10 & 10 & 10 \\
Palmiste & $5 \%$ & 50 & 50 & 50 \\
Farine de blé & $1 \%$ & 10 & 10 & 10 \\
Arachide & $4 \%$ & 40 & 40 & 40 \\
Farine d'os & $0,25 \%$ & 2,5 & 2,5 & 2,5 \\
Huile de palme & $0,50 \%$ & 5 & 5 & 5 \\
Son de blé & $0,25 \%$ & 2,5 & 2,5 & 2,5 \\
Total en Ms & $100 \%$ & 1000 & 1000 & 1000
\end{tabular}

RS = Régime Standard (contenant 10\% de la farine de poisson vendu à la provenderie) ; RA = Régime Alimentaire (substitution des $10 \%$ de la farine de poisson par la poudre des sous-produits de P.typus) ; RN = Régime Neutre (substitution des farines précédentes par les farines végétales); Ms : Matière sèche.

Tableau 2 : Paramètres d'étude de la valeur nutritionnelle.

\begin{tabular}{|c|c|}
\hline Paramètres & Expressions mathématiques \\
\hline Matière sèche totale ingérée (MSTI) & $\begin{array}{l}\text { Somme des quantités de matière sèche (de l'aliment) } \\
\text { ingérée durant la période d'expérimentation }\end{array}$ \\
\hline Protéines totales ingérées (PTI) & MSTI x pourcentage de protéines du régime \\
\hline Gain de poids (GP) & Poids final - Poids initial \\
\hline Coefficient d'Efficacité Alimentaire (CEA) & $C E A=\frac{\text { Gain de poids }(g)}{\text { Matière séche totale ingérée }(g)}$ \\
\hline Coefficient d'Efficacité Protéique (CEP) & $C E P=\frac{\text { Gain de poids }(g)}{\text { Protéine totale ingérée }(g)}$ \\
\hline $\begin{array}{l}\text { Digestibilité } \\
\text { • Apparent }\end{array}$ & $I-F$ \\
\hline - Réel & $\begin{array}{c}C U D a=\frac{I}{I} \times 100 \\
C U D r=\frac{I-(F-F s p)}{I} \times 100\end{array}$ \\
\hline Utilisation pratique de l'azote (UPN) & $U P N=\frac{I-(F-F s p)-(U-U s p)}{I} \times 100$ \\
\hline Valeur biologique (VB) & $V B=\frac{I-(F-F s p)-(U-U s p)}{I-(F-F s p)} \times 100$ \\
\hline
\end{tabular}


RESULTATS

Essai d'estimation de la quantité de sousproduits générée par le nettoyage de Pseudotolithus typus

Les résultats morpho-métriques sont consignés dans le Tableau 3. Ces résultats montrent que les poissons de l'espèce $P$. typus étaient tous matures et embonpoint.

Pour une masse totale de poissons entiers de $271 \mathrm{~kg}$, l'on a obtenu $237 \mathrm{~kg}$ de poissons éviscérés et $34 \mathrm{~kg}$ des sous-produits soit $12,55 \%$ de la masse totale des poissons entiers. Les rejets après nettoyage des viscères et branchies représentent plus de la moitié des $12,55 \%$ soit environ $18,42 \mathrm{~kg} \quad(6,8 \%)$ (Excréments, biles, reste d'aliment) et le reste $15,58 \mathrm{~kg}(5,75 \%)$ constitué de l'appareil digestif, foie, cœur, œuf et branchie. La matière sèche obtenue après séchage à l'étuve a donné $4,7 \mathrm{~kg}$ soit une différence de poids de la matière fraiche à la matière sèche de $69,83 \%$.

\section{Composition proximale des sous-produits et qualité des huiles extraites}

Les valeurs obtenues de la composition proximale sont consignées dans le Tableau 4. Ces sous-produits sont pauvres en glucides et présentent des teneurs en protéines, en cendres et en lipides importantes. Les macroéléments présentent des teneurs plus importantes en phosphore, ensuite en calcium, avec des faibles teneurs en sodium. On note aussi un bon rendement pour le rapport calcium/phosphore qui est de 0,83 et très faible pour le rapport sodium/potassium qui est de 0,23 . Quant aux microéléments, ils présentent une teneur plus importante en fer, ensuite viennent le zinc et le cuivre avec une faible teneur en manganèse.

Les valeurs obtenues des indices étudiées permettant la caractérisation des huiles extraites des sous-produits sont consignées dans le Tableau 5. Les résultats présentent une forte teneur en indice diode par rapport aux autres indices, avec l'indice anisidine qui ferme cette série avec une très faible teneur, précédé de l'indice de peroxyde.

\section{Expérimentation animale}

Les valeurs obtenues des paramètres étudiés sont consignées dans le Tableau 6. On note une différence significative à $\mathrm{p} \leq 0,05 \mathrm{du}$ régime alimentaire (RA) par rapport aux deux autres régimes ( $\mathrm{RS}$ et $\mathrm{RN}$ ) pour la matière sèche totale ingérée, la protéine totale ingérée, et pour les digestibilités apparente (CUDa) et réel (CUDr). La Figure 1 montre la variation alimentaire (Figure 1a) et la variation du gain de poids (Figure $1 b$ ) des différents régimes. De cette Figure, il ressort que les rats du lot 2 soumis au régime alimentaire consomme moins d'aliment que les rats du lot 3 soumis au régime neutre, mais présentent des gains de poids plus élevés que ceux-ci.

Tableau 3 : Moyenne de quelques Mesures morpho-métriques de l'espèce P. typus.

\begin{tabular}{ll}
\hline Morpho-metriques & Mesures \\
\hline Longueur totale moyen $(\mathrm{cm})$ & $33,95 \pm 4,990$ \\
Longueur standard moyen $(\mathrm{cm})$ & $27,0236 \pm 4,429$ \\
Poids total moyen $(\mathrm{g})$ & $323,063 \pm 146,492$ \\
& \\
Poids éviscérés moyen $(\mathrm{g})$ & $285,747 \pm 132,532$ \\
Facteur de condition $\left(\mathrm{g} / \mathrm{cm}^{\mathrm{b}}\right)$ & $0,255 \pm 0,069$ \\
Indice viscéro-somatique $\left.(\%)_{\mathrm{g} / \mathrm{cm}^{\mathrm{b}} \text { : gramme par centimètre à la puissance b. Les valeurs présentées sont des moyennes et leur écart- type }(\bar{x}, \delta}\right)$.
\end{tabular}


Tableau 4 : Composition chimique des sous-produits.

\begin{tabular}{llllll}
\hline Macronutriments & $\begin{array}{l}\text { Humidité } \\
(\% \mathbf{M s})\end{array}$ & $\begin{array}{l}\text { Protéines } \\
(\% \mathbf{M s})\end{array}$ & $\begin{array}{l}\text { Lipides } \\
(\% \mathbf{M s})\end{array}$ & $\begin{array}{l}\text { Cendres } \\
(\% \mathbf{M s})\end{array}$ & $\begin{array}{l}\text { Glucide Totaux } \\
(\% \mathbf{M s})\end{array}$ \\
\hline $\begin{array}{l}\text { Teneurs } \\
\mathrm{n}=3\end{array}$ & $20,33 \pm 0,57$ & $59,81 \pm 0,17$ & $15,11 \pm 0,05$ & $24,33 \pm 0,57$ & $0,75 \pm 0,46$ \\
\hline Macroéléments $(\mathrm{g} / \mathrm{kg})$ & $\mathrm{Ca}$ & $\mathrm{Mg}$ & $\mathrm{K}$ & $\mathrm{P}$ & $\mathrm{Na}$ \\
\hline $\begin{array}{l}\text { Teneurs } \\
\mathrm{n}=3\end{array}$ & $4,63 \pm 0,42$ & $1,32 \pm 0,18$ & $1,53 \pm 0,23$ & $5,59 \pm 0,45$ & $0,35 \pm 0,004$ \\
\hline Microéléments $(\mathrm{mg} / \mathrm{kg})$ & $\mathrm{Zn}$ & $\mathrm{Mn}$ & $\mathrm{Cu}$ & $\mathrm{Fe}$ & \\
\hline $\begin{array}{l}\text { Teneurs } \\
\mathrm{n}=3\end{array}$ & $86,15 \pm 6,81$ & $18,49 \pm 1,86$ & $44,34 \pm 4,04$ & $323 \pm 18,36$ & \\
\hline
\end{tabular}

$\%$ Ms : pourcentage Matière sèche. Les valeurs présentées sont des moyennes et leur écart- type $(\bar{x}, \delta)$

Tableau 5 : Valeur des indices étudiés.

\begin{tabular}{llllllll}
\hline Indices & $\begin{array}{l}\text { IP (mg / kg } \\
\text { d'huile) }\end{array}$ & $\begin{array}{l}\text { I.An (mg / / } \\
\text { kg d'huile) }\end{array}$ & $\begin{array}{l}\text { TOTOX (mg } \\
\text { / kg d'huile }\end{array}$ & $\begin{array}{l}\text { Ia }(\mathbf{m g} \text { KOH/ } \\
\text { kg d'huile) }\end{array}$ & $\begin{array}{l}\text { Ii (mg } \\
\text { d'huile) }\end{array}$ & / kg \\
\hline $\begin{array}{l}\text { Teneurs } \\
\mathrm{n}=2\end{array}$ & $0,52 \pm 0,02$ & $0,27 \pm 0,01$ & $1,30 \pm 0,04$ & $49,95 \pm 1,17$ & $122,74 \pm 0,92$ & \\
\hline
\end{tabular}

IP : Indice de peroxyde ; I.An : Indice d'anisidine ; TOTOX : Oxydation totale ; Ia : Indice d'acide ; Ii : Indice d'iode. Les valeurs présentées sont des moyennes et leur écart- type $(\bar{x}, \delta)$.

Tableau 6 : Valeurs des paramètres étudiés.

\begin{tabular}{llllll}
\hline Régimes & MSTI $(\mathbf{g} / \mathbf{j})$ & PTI $(\mathbf{g} / \mathbf{j})$ & GP $(\mathbf{g} / \mathbf{j})$ & CEA & CEP \\
\hline RA & $18,59 \pm 1,24^{\mathrm{a}}$ & $1,11 \pm 0,07^{\mathrm{a}}$ & $1,63 \pm 0,49^{\mathrm{a}}$ & $0,087 \pm 0,021^{\mathrm{a}}$ & $1,453 \pm 0,344^{\mathrm{a}}$ \\
RS & $22,24 \pm 0,29^{\mathrm{b}}$ & $1,36 \pm 0,02^{\mathrm{b}}$ & $2,19 \pm 0,16^{\mathrm{a}}$ & $0,098 \pm 0,006^{\mathrm{a}}$ & $1,606 \pm 0,097^{\mathrm{a}}$ \\
RN & $21,48 \pm 0,28^{\mathrm{b}}$ & 0 & $1,58 \pm 0,50^{\mathrm{a}}$ & $0,075 \pm 0,029^{\mathrm{a}}$ & $/$ \\
\hline
\end{tabular}

\begin{tabular}{lllll}
\hline Régimes & CUDa (\%) & CUDr (\%) & UPN (\%) & VB (\%) \\
\hline RA & $99,52 \pm 0,01^{\mathrm{a}}$ & $100,34 \pm 0,19^{\mathrm{a}}$ & $99,45 \pm 1,16^{\mathrm{a}}$ & $99,47 \pm 0,68^{\mathrm{a}}$ \\
\hline RS & $99,33 \pm 0,053^{\mathrm{b}}$ & $99,99 \pm 0,15^{\mathrm{b}}$ & $99,96 \pm 0,81^{\mathrm{a}}$ & $99,98 \pm 0,41^{\mathrm{a}}$
\end{tabular}

MSTI : Matière sèche totale ingérée ; PTI : Protéines totales ingérées; GP : Gain de poids ; CEA : Coefficient d'Efficacité Alimentaire ; CEP : Coefficient d'Efficacité Protéique ; CUDa : Digestibilité apparent ; CUDr : Digestibilité réel ; UPN : Utilisation pratique de l'azote ; VB : Valeur biologique ; RS = Régime Standard (contenant $10 \%$ de la farine de poisson vendu à la provenderie) ; RA = Régime Alimentaire (substitution des $10 \%$ de la farine de poisson par la poudre des sous-produits de P.typus) $; \mathrm{RN}=$ Régime Neutre (substitution des farines précédentes par les farines végétales). Chaque valeur est la moyenne \pm écart-type $(\bar{x}, \delta)$ des six rats. Il n'y a aucune différence significative $(\mathrm{p} \leq 0,05)$ entre deux moyennes \pm écart-type (à l'intérieur de la même colonne) désignées par la même lettre. 
Variation alimentaire

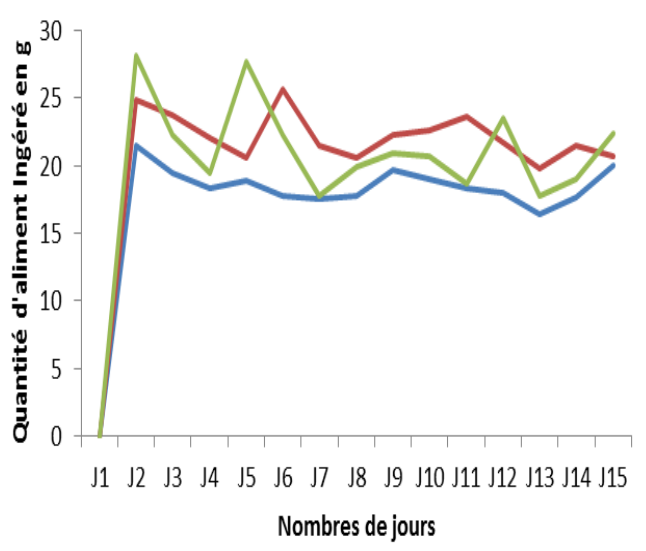

Figure 1a : Variation alimentaire.

\section{Variation du Gain de Poids}
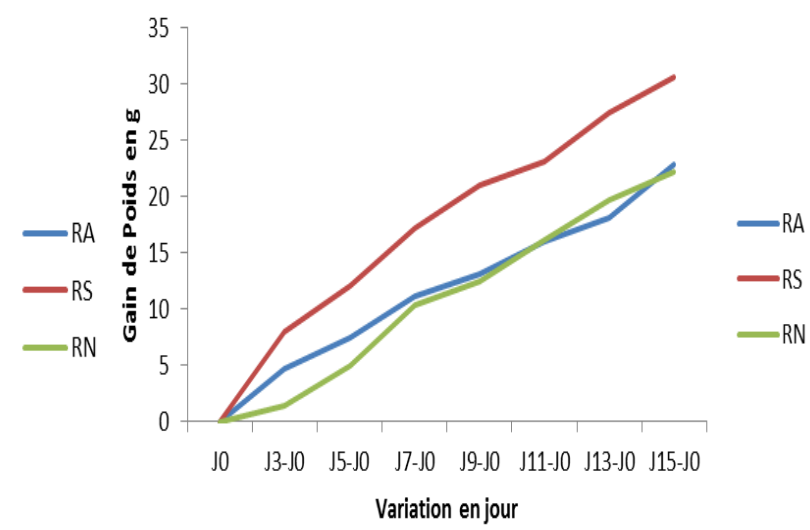

Figure 1b : Variation du Gain de poids.

Figure 1 : Variation alimentaire et variation du gain de poids.

RS = Régime Standard (contenant 10\% de la farine de poisson vendu à la provenderie) ; RA = Régime Alimentaire (substitution des $10 \%$ de la farine de poisson par la poudre des sous-produits de P.typus); RN = Régime Neutre (substitution des farines précédentes par les farines végétales).

\section{DISCUSSION}

Le pourcentage des sous-produits obtenus (12,55\%) par rapport au poids total des espèces utilisées reste dans l'intervalle du résultat obtenu par Le Floc'h et al. (2004), le pourcentage étant de $12-18 \%$ du poisson entier et servant à la production de la farine de poisson, comme aliment, production d'huile et enzyme.

Une comparaison entre les sousproduits utilisés et la partie comestible montre que les teneurs en protéine, calcium, magnésium et en zinc des sous-produits sont inférieures à celles de la partie comestible (le filet) de P. typus. Par contre les teneurs en lipide, en matière sèche, en cendre et quelques minéraux $(\mathrm{P}, \mathrm{K}, \mathrm{Na}, \mathrm{Fe})$ des sous-produits, sont supérieures à celles de la partie comestible de P. typus (Njinkoue et al., 2016).

Les teneurs en fer des sous-produits de $P$. typus sont supérieures aux résultats obtenus par Njinkoue et al. (2016) dans les parties comestibles de Pseudotolitus typus, de Pseudotolitus elongatus et Pseudotolitus senegalensis, et des teneurs inférieures en calcium et en phosphore pour les mêmes espèces. Les mêmes résultats sont constatés sur le filet de $P$. senegalensis où les protéines restent supérieures à celles des sous-produits de $P$. typus et les autres éléments nutritifs sont inférieurs (Nganguem, 2007). Les teneurs en protéines, cendres, potassium et calcium des sous-produits de l'espèce de $P$. typus sont supérieures à celles de la farine d'asticots séchés et les teneurs en lipides et sodium sont plutôt inférieures. En plus, les teneurs en lipides, en cendres et en potassium des sousproduits issus de $P$. typus sont supérieures à celles de la farine de poisson et les teneurs en protéines, calcium et sodium sont plutôt inférieures (Bouafou, 2007).

L'huile des sous-produits des poissons issus des poissons de l'espèce de $P$. typus a un indice de peroxyde proche de celui recommandé par l'homologation qui doit être inférieur ou égale à 5 milliéquivalents d'oxygène actif/kg d'huile (Codex Alimentarius, 2017). La valeur de p-anisidine 
obtenue est inférieure à celle préconisée par le Codex Alimentarius $\leq 20$ méq $/ \mathrm{kg}$ d'huile pour les huiles de poisson. Pour l'oxydation totale la valeur obtenue est inférieure à celle préconisée par le Codex Alimentarius $\leq 26$ méq $/ \mathrm{kg}$ d'huile pour les huiles de poisson. L'indice d'acide obtenu est proche de celui recommandé par la norme, qui doit être inférieur ou égale à $3 \mathrm{mg}$ $\mathrm{KOH} / \mathrm{g}$ (Codex Alimentarius, 2017).

L'indice d'iode de cette huile est élevé, cette valeur présente les sous-produits des poissons de l'espèce $P$. typus comme riche en acides gras polyinsaturés. En effets les travaux menés par Njinkoue et al. (2016) ont montré que les acides gras polyinsaturés w3 et w6 représentent $33,9 \%$ des acides gras totaux contenu dans l'huile de P. typus.

Les rats du lot 2 soumis au régime RA consomment moins d'aliment que les rats du lot 1 et 3 respectivement soumis aux régimes RS et RN ; ceci peut être dû à l'appréciation de l'aliment par les rats ou aux différents constituants de l'aliment à satisfaire les besoins optimum des rats. La différence observée entre la matière sèche totale ingérée et le gain de poids pour les rats du régime RA et ceux du régime $\mathrm{RN}$ peut s'expliquer par la présence des éléments nutritifs des sous-produits susceptibles de créer cette différence. La capacité de supplémentation mutuelle entre deux ou plusieurs sources de protéines dépend de leur équilibre en acides aminés indispensables, de sorte que, la combinaison résultante peut acquérir, par le jeu des compensations mutuelles entre acides aminés indispensables (AAI) limitant et en excès, une valeur nutritionnelle qui surpasse celles de chacune des sources protéiques (Apfelbaum et al., 2004).

La différence significative à $\mathrm{p} \leq 0,05$ des digestibilités entre les régimes RS et RA par les rats du lot 1 et du lot 2 montre que les rats de chaque régime ont des digestibilités différentes. Ces résultats présentent une digestibilité plus élevée pour le régime RA par rapport au régime RS. Ceci montre que les sous-produits issus des poissons de l'espèce Pseudotolithus typus contribuent à une bonne assimilation du régime alimentaire par les rats contrairement à la farine du poisson. Ceci peut se traduire par une réduction de la disponibilité des acides aminés indispensables requis pour satisfaire les besoins de croissance optimum des rats soumis au régime RS (Meite et al., 2008). Cette information peut suggérer qu'à fort taux dans le régime, les protéines de la farine de poisson sont difficilement dégradables par les différentes enzymes digestives dans l'organisme (Bouafou et al., 2011).

\section{Conclusion}

L'étude de la composition chimique et la valeur nutritionnelle des viscères et branchies issus du nettoyage du poisson de l'espèce Pseudotolithus typus, montre que ces sous-produits de P.typus sont riches en macronutriments, en macroéléments et en microéléments. Les huiles extraites sont de bonne qualité. Le régime alimentaire est plus digeste par rapport au régime standard. Les rats $\mathrm{du}$ régime alimentaire consomment moins d'aliment que les rats du régime neutre, et ont un gain de poids moyen supérieur à ceux-ci. Ces viscères et branchies de $P$. typus ont un potentiel nutritionnel élevé et peuvent être utilisés comme complément alimentaire pour bétail.

\section{CONFLITS D'INTERETS}

Les auteurs déclarent ne pas avoir de conflits d'intérêts en relation avec ce manuscrit.

\section{CONTRIBUTIONS DES AUTEURS}

$\mathrm{JMN}$ : Initiateur du thème, rédaction et interprétation et auteur de correspondance; PRNN: Elaboration du protocole de travail, travaux au laboratoire, à l'animalerie et sur la paillasse, rédaction et interprétation des 
résultats ; JCMK : Travaux à l'animalerie et sur la paillasse; CSMM : Participation au travail de paillasse; JBD: Participation au travail de paillasse ; MNN : Participation au travail de paillasse; SRM : Analyse des données et relecture du travail; FT: Responsable d'équipe et supervision du travail.

\section{REMERCIEMENTS}

Nous remercions tous les nettoyeurs des produits halieutiques au marché Central de New-bell dans l'arrondissement de Douala II ; le Laboratoire des Sciences Alimentaires et Nutrition de la Faculté des Sciences, et le Laboratoire des Ecosystèmes et des Ressources Halieutiques, de l'Université de Douala ; et Le Ministère de l'Enseignement Supérieur du Cameroun au travers de l'Appui à la Modernisation de la Recherche.

\section{REFERENCES}

AFNOR. Association Française de Normalisation. 1981. Recueil des Normes Françaises. Corps gras, graines oléagineuses, produits dérivés (2ème édition). AFNOR : Paris, France ; 438p.

Anaïs P. 2014. La gestion des ressources et des territoires : Application à la mise en œuvre de projets de valorisation de sousproduits de poisson, 394p.

AOAC (Association of Official Analytical Chemist). 1990. Official Method of Analysis (15 $5^{\text {th }}$ edn). AOAC: Washington DC ; 222-245.

Apfelbaum M, Romon M, Dubus M. 2004. Diététique et Nutrition. (6è édn). Masson Ed : Paris ; 38-45.

Bouafou KGM, Konan BA, Meite A, Kouame KG, Katy-Coulibally S. 2011. Détermination du taux optimal de farine d'asticots séchés dans le régime du rat en croissance Journal of Animal \& Plant Sciences, 12(2): 1553-1559. DOI: http://www.m.elewa.org/JAPS/2011/12. 2/2.pdf
Bouafou KGM. 2007. Etude de la production d'asticots à partir d'ordures ménagères et de la valeur nutritionnelle de la farine d'asticots séchés (FAS) chez le rat en croissance. Thèse Doct. Physiol. Anim., Université de Cocody, Abidjan, Côte d'Ivoire, p. 145.

Commission du Codex Alimentarius. 2017. Programme mixte FAO/OMS sur les normes alimentaires. Rapport de la vingtcinquième session du comité du Codex sur les graisses et les huiles.Kuala Lumpur (Malaisie). 16-28.

FAO. 2016. Organisation des nations unies pour l'agriculture. La situation mondiale de la pêche et de l'aquaculture. Rome. $241 \mathrm{p}$.

Khalifa SBS. 2011. Valorisation de co-produits de la sole tropicale (Cynoglossus senegalensis) par hydrolyse enzymatique: Application en nutrition avicole, $119 \mathrm{p}$. DOI : https://archimer.ifremer.fr/doc/00057/16 788/14240.pdf

Le Floc'h $\mathrm{P}$, Bourseau $\mathrm{P}$, Daurès F, Guérard F, Le Grel L, Meunier M, Tuncel M. 2004. Valorisation des coproduits de la mer et territoire : enjeux territoriaux, Classification JEL : C81, Q22, R11. DOI :

https://www.researchgate.net/publication /227382191

Meite A, Kouame KG, Kati-coulibaly S, Offoumou MA. 2008. Étude de la valeur nutritionnelle du pain normal et des pains composites contenant de la farine de graines délipidées de Citrullus lanatus (Cucurbitacées) Laboratoire Nutrition et Pharmacologie, UFR Biosciences, Université de Cocody, 22 BP 582 Abidjan 22, Abidjan, Côte d'Ivoire. DOI : $\quad$ https://popups.uliege.be/00379565/index.php?id=595

Nganguem M. 2007. Approche physicochimique du pouvoir conservateur du sel: 
Cas du salage de Pseudotolithus senegalensis. Thèse de Maîtrise Professionnelle de Biotechnologie, Université d'Abomey-Calavi. Cotonou, Niger.

DOI : https://www.memoireonline.com/02/08/ 940/approche-physico-chimique- ouvoirconservateur-sel-pseudotolithusenegalensis.html

Njinkoue JM, Gouado I, Tchounbougnang F, Yanga JH, Ngueguim, Ndinteh DT, Fodjo CYF, Schweigert FJ. 2016. Proximate composition, mineral content and fatty acid profile of two marine fisches form cameroonian coast: Pseudotolithus typus(Bleeker, 1863) and Pseudotolithus elongatus (Bowdich,
1825). NFS Journal, 4(2016): 27-31. DOI :

http://dx.doi.org/10.1016/j.nfs.2016.07.0 02.

Shahidi F, Miraliakbari H. 2008. Antioxidant activity of minor components of tree nut oils. Food Chemistry, 111: 421-427. DOI:

http://dx.doi.org/10.1016/j.foodchem.20 08.04.008

UIPAC (International Union of Pure and Applied Chemistry). 1981. Stantard Methods for the Analysis of Oils, Fats and Derivates $\left(6^{\text {th }}\right.$ Edn). UIPAC; 646p. 